\title{
Research on Temperature Compensation Algorithm in Torque Sensor of E-bike
}

\author{
Xiadong Zhang ${ }^{1, ~ a}$, Yunfei $\mathrm{Li}^{1, \mathrm{~b}}$ and Juncheng Jia ${ }^{1, \mathrm{c}}$ \\ ${ }^{1}$ School of Computer Science and Technology, Soochow University, Suzhou 215000, China; \\ a515246437@qq.com
}

Keywords: Torque sensor; Surface fitting; Least-square method; NRF24LE1.

\begin{abstract}
When using torque sensor consisting of resistance strain gauge for the torque measurement in the E-bike, there is a nonlinear problem affected by temperature. In order to solve it, the surface fitting algorithm based on least-square method is proposed in this paper. Based on the error analysis of the torque sensor and the feasibility analysis of the system, the surface fitting algorithm is designed and implemented in the microprocessor NRF24LE1. The test results indicate that the algorithm works well with high stability and accuracy. The algorithm has great value of application with significant compensation effect, which provides a technical selection for the development and improvement of the E-bike.
\end{abstract}

\section{Introduction}

With the increment of consciousness for environmental protection and energy-saving, the E-bike (electric bicycle) based on torque sensor has replayed the traditional electric bicycle and occupied a certain market [1]. As a core component, torque sensor is the key of research for the E-bike. Some torque sensors like hull, crankshaft and resistance strain gauge have been used in torque sensing system of the E-bike [2]. Comparing with the others, the torque sensor based on the resistance strain gauge with high sensitivity and small volume shows significant advantages. However, the output of the torque sensor based on the resistance strain gauge is influenced by the temperature and exists nonlinearity. The nonlinearity rectification and temperature compensation algorithm are used to improve the accuracy of the sensor [3].

In this paper, the values of the input torque, working temperature and output voltage are used as a variable. The model of the surface fitting for the sensor is built up by the least-square method. Once the values of the input torque, working temperature is acquired, the value of the torque would be calculated by using the model.

\section{Error analysis of the torque sensor}

The resistance-strain effect is utilized in the torque sensor. Four strain gauges are pasted on the specified position of the axis and a Wheatstone bridge is built up. And a tiny stress change on the axis can result in the change of the balance status of the bridge. As a result, the amount of the resistance change can be converted into $\mathrm{mV}$ level by Wheatstone bridge [4]. Since the output signal of torque sensor is $\mathrm{mV}$ level and is hardly measured, the amplification circuit and A/D conversion circuit should be incorporated. The sensor measure circuit is shown in Figure 1.

Two factors result in the main error of the torque sensor. The first one is the nonlinear error of the resistance strain gauge and the Wheatstone bridge. The second is the influence of the temperature changes. Due to the two factors mentioned above, the zero output of the torque sensor occurs when the E-bike is working, which brings the phenomenon of the self-starting. Moreover, because the output value of the torque sensor is influenced by the temperature, the E-bike raises different performance when the temperature changes. 


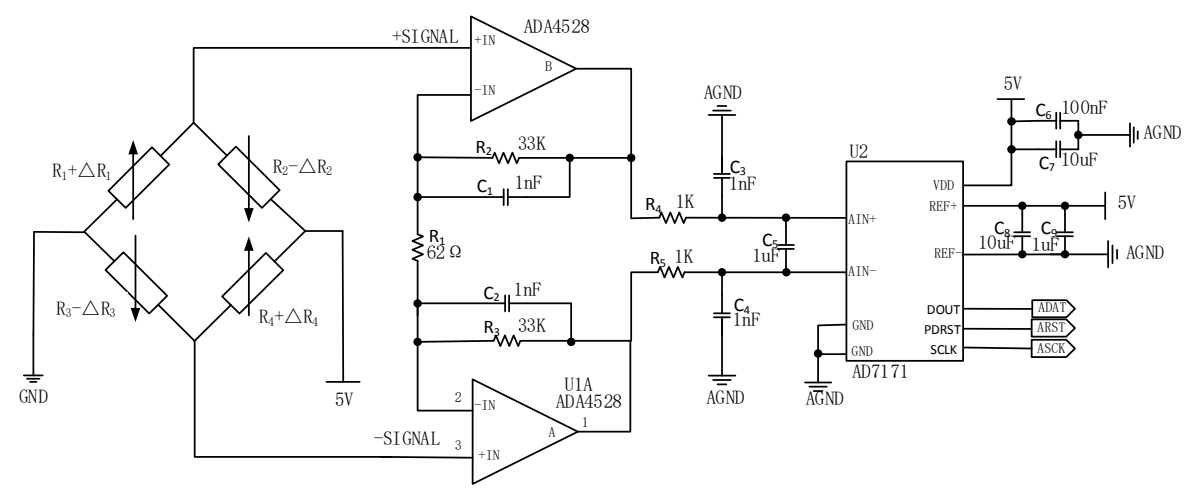

Figure 1 The sensor measure circuit

\section{The nonlinearity rectification and temperature compensation algorithm}

In this paper, to solve the problem which is analyzed above, the algorithm based on the surface fitting of the least-square method is proposed.

\subsection{The output characteristic of the torque sensor}

To describe the output characteristic of the torque sensor, two torque sensors of the E-bike are tested in different conditions. In test experiment, every sensor is set in the temperature control box. The output of the torque sensor is recorded and shown in Figure 2.
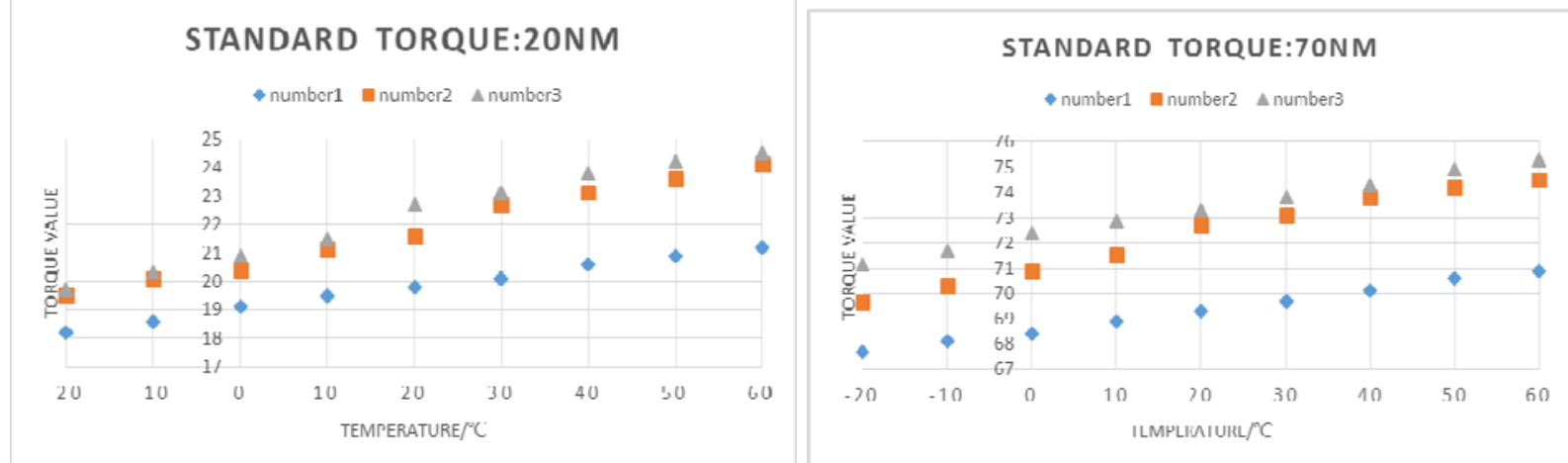

Figure 2 The output characteristic of the torque sensor

As shown in Figure 2, the torque sensors called number1, number2 and number3 are tested in the experiment, and the output of the torque sensors increase with temperature in the range of $-20 \sim 60^{\circ} \mathrm{C}$. Different torque sensors show different characteristics.

\subsection{The description of the algorithm}

The nonlinearity rectification and temperature compensation is implement by the fitting of the software. The relationship of the standard torque $(\mathrm{M})$, the original torque $\left(\mathrm{M}_{\mathrm{p}}\right)$ and the temperature $\left(T_{\mathrm{p}}\right)$ can be expressed as follow,

$$
M=f\left(M_{p}, T_{p}\right)
$$

The formula (1) is expanded by the Taylor's series in point $\left(\mathrm{M}_{\mathrm{p} 0}, \mathrm{~T}_{\mathrm{p} 0}\right)$, and the results can be expressed as follow,

$$
\begin{aligned}
f\left(M_{p}, T_{p}\right)= & f\left(M_{p 0}, T_{p 0}\right)+\frac{1}{1 !}\left[\left(M_{p}-M_{p 0}\right) \frac{\partial}{\partial M_{p}}+\left(T_{p}-T_{p 0}\right) \frac{\partial}{\partial T_{p}}\right] f\left(M_{p 0}, T_{p 0}\right) \\
& +\frac{1}{2 !}\left[\left(M_{p}-M_{p 0}\right) \frac{\partial}{\partial M_{p}}+\left(T_{p}-T_{p 0}\right) \frac{\partial}{\partial T_{p}}\right]^{2} f\left(M_{p 0}, T_{p 0}\right)+\ldots . \\
& +\frac{1}{n !}\left[\left(M_{p}-M_{p 0}\right) \frac{\partial}{\partial M_{p}}+\left(T_{p}-T_{p 0}\right) \frac{\partial}{\partial T_{p}}\right]^{n} f\left(M_{p 0}, T_{p 0}\right)+R_{n}
\end{aligned}
$$

where the $R_{n}$ is the Lagrange remainder. $R_{n}$ can be expressed as follow, 


$$
R_{n}=\frac{1}{(n+1) !}\left[\left(M_{p}-M_{p 0}\right) \frac{\partial}{\partial M_{p}}+\left(T_{p}-T_{p 0}\right) \frac{\partial}{\partial T_{p}}\right]^{n+1} f\left((1-\theta) M_{p 0}+\theta M_{p},(1-\theta) T_{p 0}+\theta T_{p}\right), 0<\theta<1
$$

Due to the accuracy of the data, the formula (2) is taken to the quadratic term. The formula (2) can be simplified as follow,

$$
f\left(M_{p}, T_{p}\right)=k_{00}+k_{10} M_{p}+k_{01} T_{p}+k_{20} M_{p}^{2}+k_{11} M_{p} T_{p}+k_{02} T_{p}{ }^{2}+k_{21} M_{p}^{2} T_{p}+k_{12} M_{p} T_{p}^{2}+k_{22} M_{p}^{2} T_{p}^{2}
$$

where $\mathrm{k}_{00}, \mathrm{k}_{01}, \mathrm{k}_{10}, \mathrm{k}_{20}, \mathrm{k}_{11}, \mathrm{k}_{21}, \mathrm{k}_{12}, \mathrm{k}_{22}$ are the coefficient. The data of $\mathrm{M}_{\mathrm{p}}, \mathrm{T}_{\mathrm{p}}$ and $\mathrm{M}$ is obtained by the acquisition experiment. The acquisition experiment is implement by following these steps:

Step 1: The temperature change is implemented by the temperature control box. The standard torque is exerted by the torque wrench.

Step 2: The working temperature-point is set every $5^{\circ} \mathrm{C}$. The range of the experiment temperature is $-20 \sim+60^{\circ} \mathrm{C}$.

Step 3: In every working temperature-point, the torque wrench is set every $5 \mathrm{NM}$. The range of the torque is $0 \sim 80 \mathrm{NM}$.

Step 4: The data obtained in above steps is constructed the surface fitting model (formula (4)).

Step 5: The coefficient of the formula (4) is stored in the FLUSH of the microprocessor.

\section{The testing methods, results and analysis}

\subsection{The choice of the microprocessor}

The NRF24LE1 microprocessor with lower power consumption and $2.4 \mathrm{GHz}$ transceivers is selected as the core component to implement the fitting algorithm. The parameter $\mathrm{k}_{00}, \mathrm{k}_{01}, \mathrm{k}_{10}, \mathrm{k}_{20}, \mathrm{k}_{11}, \mathrm{k}_{21}$, $\mathrm{k}_{12}, \mathrm{k}_{22}$ are stored in the FLASH of the microprocessor. The value of torque can be calculated by (4) after the voltage signal is obtained by the A/D conversion circuit. The schematic diagram of the minimum system is showed in Figure 3.

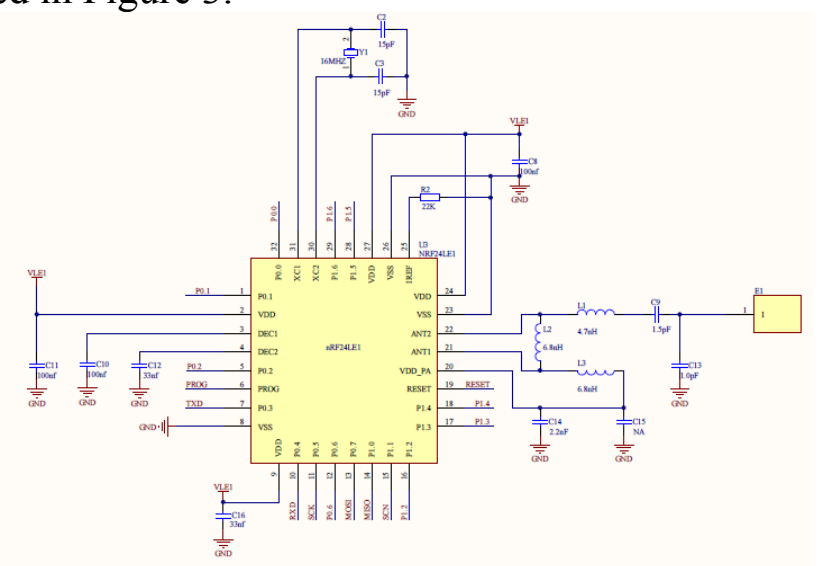

Figure 3 The schematic diagram of the minimum system

\subsection{The testing methods and results}

The hardware system of the test platform includes gear and chain, servo motor, standard torque sensor and magnetic powder brake. The servo motor drives gear and chain to simulate the actual torque during riding. The magnetic powder brake provides the load to simulate the resistance. The standard torque sensor is used to provide standard torque to compare with the measured torque signal. The test platform is put into the temperature control box, three torque test points $(20 \mathrm{NM}, 40 \mathrm{NM}$, $60 \mathrm{NM}, 80 \mathrm{NM}$ ) are set and nine temperature test points are set every $10^{\circ} \mathrm{C}$. The testing results are showed in Table1, 2, 3, 4.

Table 1 Standard Torque of 20NM

\begin{tabular}{cccccccccc}
\hline Temperature & -20 & -10 & 0 & 10 & 20 & 30 & 40 & 50 & 60 \\
\hline Torque & 19.79 & 19.82 & 19.85 & 19.78 & 19.77 & 19.75 & 19.81 & 19.74 & 19.77 \\
Error/NM & 0.21 & 0.18 & 0.15 & 0.22 & 0.23 & 0.25 & 0.19 & 0.26 & 0.23 \\
\hline
\end{tabular}


Table 2 Standard Torque of 40NM

\begin{tabular}{cccccccccc}
\hline Temperature & -20 & -10 & 0 & 10 & 20 & 30 & 40 & 50 & 60 \\
\hline Torque & 40.26 & 40.18 & 40.14 & 40.21 & 40.28 & 40.29 & 40.14 & 40.26 & 40.23 \\
Error/NM & 0.26 & 0.18 & 0.14 & 0.21 & 0.28 & 0.29 & 0.14 & 0.26 & 0.23 \\
\hline \multicolumn{8}{c}{ Table 3 Standard Torque of $60 \mathrm{NM}$} \\
\hline Temperature & -20 & -10 & 0 & 10 & 20 & 30 & 40 & 50 & 60 \\
\hline Torque & 60.31 & 60.29 & 60.27 & 60.24 & 60.23 & 60.25 & 60.27 & 60.29 & 60.23 \\
Error/NM & 0.31 & 0.29 & 0.27 & 0.24 & 0.23 & 0.25 & 0.27 & 0.29 & 0.23 \\
\hline \multicolumn{8}{c}{ Table } & 4 Standard Torque of $80 \mathrm{NM}$ & \\
\hline Temperature & -20 & -10 & 0 & 10 & 20 & 30 & 40 & 50 & 60 \\
\hline Torque & 80.29 & 80.24 & 80.19 & 80.22 & 80.23 & 80.28 & 80.21 & 80.26 & 80.23 \\
Error/NM & 0.29 & 0.24 & 0.19 & 0.22 & 0.23 & 0.28 & 0.21 & 0.26 & 0.23 \\
\hline
\end{tabular}

\subsection{The analysis of the results}

According to the testing results, the surface fitting algorithm reduces the influence of the temperature and improve the data accuracy. The testing dada shows the range of the full scale error is no more than $0.6 \% \mathrm{FS}$, which fully satisfied the actual requirement.

\section{Conclusion}

In this paper, to compensate the influence of the temperature to the torque sensor of the E-bike, a surface fitting algorithm based on least-square method is proposed. Based on the feasibility analysis for the algorithm, the algorithm is implemented in the microprocessor NRF24LE1. The test results indicate that the algorithm works well with high stability and accuracy. On the other hand, it satisfies the application requirement and provides a technical selection for the development and improvement of the E-bike.

\section{ACKNOWLEDGMENT}

This work is supported in part by National Natural Science Foundation of China (61272449), Joint Innovation Funding of Jiangsu Province (BY2014059-02), and Collaborative Innovation Center of Novel Software Technology and Industrialization.

\section{References}

[1]Wikipedia. Electric bicycle [EB/OL]. 2012-11-19. http: //en. wikipedia.org /Wikipedia.org /wiki/ Electric_bicycle.

[2]ID bike. TMM sensor uint [EB/ OL]. 2012-01. http : // www. idbike. com/ tmm-powermanagement. htm.

[3]Thun. Sensor technology: The sensory inner bearings [EB/OL]. 2010-05-09. http : // www. thun. de/ en / products /sensor -technology..

[4]INAGAKI N. Theory of image impedance matching for inductively coupled power transfer system [J]. Microwave Theory and Techniques. 2014,62(4):901-908. 